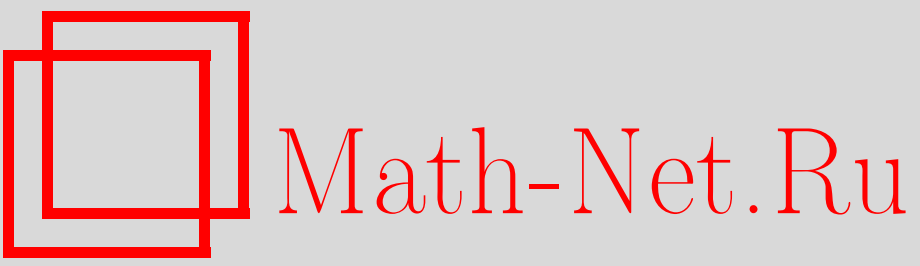

К. Аторн, Ковариантные гиперэллиптические функции рода 2, ТМФ, 2003, том 137, номер 1, 5-13

DOI: https://doi.org/10.4213/tmf238

Использование Общероссийского математического портала Math-Net.Ru подразумевает, что вы прочитали и согласны с пользовательским соглашением

http://www . mathnet.ru/rus/agreement

Параметры загрузки:

IP: 54.164 .48 .24

26 апреля 2023 г., 15:22:27 


\section{КОВАРИАНТНЫЕ ГИПЕРЭЛЛИПТИЧЕСКИЕ ФУНКЦИИ РОДА 2}

Излагаются результаты исследований, касающихся роли представлений группы $S L_{2}(\mathbb{C})$ в теории гиперэллиптических функций рода 2 . Обсуждается роль этих представлений как в классической теории, так и при введении семейства новых естественно ковариантных $\mathcal{P}$-функций.

Ключевые слова: гиперэллиптические кривые, ковариантность, ю-функции, производные Хироты.

Данная статья содержит краткое изложение результатов исследований, проведенных совместно с Эйлбеком и Энольски и касающихся роли представлений группы $S L_{2}(\mathbb{C})$ в теории гиперэллиптических функций второго рода. Полное рассмотрение этого вопроса можно найти в работах [1], [2]. Отправной точкой наших исследований по гиперэллиптическим функциям на кривых рода 2 является система уравнений, появившаяся в работе Бейкера 1907 г. (см. [3], с. 49). Это система из пяти дифференциальных уравнений четвертого порядка в частных производных с двумя независимыми переменными $u_{1}$ и $u_{2}$, ей удовлетворяет функция $\wp$, которую следует рассматривать как аналог $\wp$-функции Вейерштрасса для эллиптической кривой:

$$
\begin{aligned}
-\frac{1}{3} \wp_{2222}+2 \wp_{22}^{2} & =g_{2} g_{6}-4 g_{3} g_{5}+3 g_{4}^{2}+g_{4} \wp_{22}-2 g_{5} \wp_{12}+g_{6} \wp_{11}, \\
-\frac{1}{3} \wp_{2221}+2 \wp_{22} \wp_{12} & =\frac{1}{2}\left(g_{1} g_{6}-3 g_{2} g_{5}+2 g_{3} g_{4}\right)+g_{3} \wp_{22}-2 g_{4} \wp_{12}+g_{5} \wp_{11}, \\
-\frac{1}{3} \wp_{2211}+\frac{2}{3} \wp_{22} \wp_{11}+\frac{4}{3} \wp_{12}^{2} & =\frac{1}{6}\left(g_{0} g_{6}-9 g_{2} g_{5}+8 g_{3}^{2}\right)+g_{2} \wp_{22}-2 g_{3} \wp_{12}+g_{4} \wp_{11}, \\
-\frac{1}{3} \wp_{2111}+2 \wp_{21} \wp_{11} & =\frac{1}{2}\left(g_{0} g_{5}-3 g_{1} g_{4}+2 g_{2} g_{3}\right)+g_{1} \wp_{22}-2 g_{2} \wp_{12}+g_{3} \wp_{11}, \\
-\frac{1}{3} \wp_{1111}+2 \wp_{11}^{2} & =g_{0} g_{4}-4 g_{1} g_{3}+3 g_{2}^{2}+g_{0} \wp_{22}-2 g_{1} \wp_{12}+g_{2} \wp_{11} .
\end{aligned}
$$

Здесь нижние индексы обозначают частные производные по независимым переменным, а параметры $g_{i}$ представляют собой коэффициенты уравнения алгебраической кривой рода 2

$$
y^{2}=g_{6} x^{6}+6 g_{5} x^{5}+15 g_{4} x^{4}+20 g_{3} x^{3}+15 g_{2} x^{2}+6 g_{1} x+g_{0} .
$$

* Department of Mathmatics, University of Glasgow, Glasgow, G12 8QW UK. E-mail: c.athorne@maths.gla.ac.uk 
Наше исследование отчасти мотивировано наблюдением Бейкера: левые части вышеприведенных уравнений, как легко видеть, представляют собой пределы при $u_{i} \rightarrow u_{i}^{\prime}$, $i=1,2$, выражений

$$
-\frac{1}{2 \sigma\left(u_{1}, u_{2}\right)^{2}} \Delta_{\lambda} \Delta_{\mu} \Delta_{\nu} \Delta_{\rho} \sigma\left(u_{1}, u_{2}\right) \sigma\left(u_{1}^{\prime}, u_{2}^{\prime}\right)
$$

где $\Delta_{i}=\partial / \partial u_{i}-\partial / \partial u_{i}^{\prime}$ при $i=1,2$ и $\wp=-\ln \sigma$. Бейкер использовал эти выражения чисто формально для сокрашения обозначений, но современньй читатель узнает в них производные Хироты [4]. Недавние работы [5]-[8] продемонстрировали роль отображений типа Хироты в теории представлений группы $S L_{2}(\mathbb{C}) ;$ возможно, такой подход окажется полезным для понимания уравнений Бейкера.

В этой работе мы изложим соответствующую теорию представлений и те ее следствия для ковариантности уравнений Бейкера, которые объясняют роль отображений Хироты-Бейкера, а также предложим альтернативное определение фундаментальных функций ю-типа, которые более естественны и удобны с точки зрения теории представлений.

Начнем с кривой $(1)$, которую назовем кривой $\mathcal{V}$. Семейство таких кривых представляет собой семимерное пространство, точки которого переставляются при отображени$\mathrm{gX}$

$$
\begin{aligned}
& x \mapsto X=\frac{\alpha x+\beta}{\gamma x+\delta}, \\
& y \mapsto Y=\frac{y}{(\gamma x+\delta)^{3}}
\end{aligned}
$$

в том смысле, что такие преобразования переводят кривую $\mathcal{V}$ в кривую того же семейства, но, вообше говоря, с другим набором коэффициентов:

$$
Y^{2}=G_{6} X^{6}+6 G_{5} X^{5}+15 G_{4} X^{4}+20 G_{3} X^{3}+15 G_{2} X^{2}+6 G_{1} X+G_{0}
$$

Можно принять, что в (2) $\alpha \delta-\beta \gamma=1$, и, следовательно, данное отображение есть элемент $P S L_{2}(\mathbb{C})$. Будем считать, что оно индуцировано преобразованием из $S L_{2}(\mathbb{C})$

$$
\left(\begin{array}{l}
z_{1} \\
z_{2}
\end{array}\right) \mapsto\left(\begin{array}{l}
Z_{1} \\
Z_{2}
\end{array}\right)=\left(\begin{array}{cc}
\delta & \gamma \\
\beta & \alpha
\end{array}\right)\left(\begin{array}{l}
z_{1} \\
z_{2}
\end{array}\right)
$$

если произвести отождествление $x=z_{2} / z_{1}, X=Z_{2} / Z_{1}$. Примем это действие группы $S L_{2}(\mathbb{C})$ в качестве базисного представления и назовем его $V$. Тогда представление группы $S L_{2}(\mathbb{C})$, реализуемое коэффициентами уравнения кривой шестого порядка

$$
g_{i} \mapsto G_{i}=G_{i}\left(g_{0}, g_{1}, \ldots, g_{6} ; \alpha, \beta, \gamma, \delta\right),
$$

есть $\operatorname{Sym}^{6}\left(V^{*}\right)$, где $V^{*}$ - представление, дуальное $V$. 
В инфинитезимальной форме эти преобразования задаются векторными полями на девятимерном пространстве переменных и параметров $\left(x, y, g_{6}, g_{5}, g_{4}, g_{3}, g_{2}, g_{1}, g_{0}\right)$ :

$$
\begin{aligned}
& e=-\frac{\partial}{\partial x}+\sum_{p=0}^{6}(6-p) g_{p+1} \frac{\partial}{\partial g_{p}}, \\
& f=x^{2} \frac{\partial}{\partial x}+3 x y \frac{\partial}{\partial y}+\sum_{p=0}^{6} p g_{p-1} \frac{\partial}{\partial g_{p}}, \\
& h=-2 x \frac{\partial}{\partial x}+3 y \frac{\partial}{\partial y}+\sum_{p=0}^{6}(2 p-6) g_{p} \frac{\partial}{\partial g_{p}},
\end{aligned}
$$

которые реализуют представление алгебры $s l_{2}(\mathbb{C})$ :

$$
[h, e]=2 e, \quad[h, f]=-2 f, \quad[e, f]=h .
$$

Ковариантность кривой $\mathcal{V}$ выражается следующими утверждениями:

$$
\begin{gathered}
e\left(y^{2}-g(x)\right)=0, \\
f\left(y^{2}-g(x)\right)=6 x\left(y^{2}-g(x)\right), \\
h\left(y^{2}-g(x)\right)=6\left(y^{2}-g(x)\right),
\end{gathered}
$$

где $g(x)$ есть полином

$$
g_{6} x^{6}+6 g_{5} x^{5}+15 g_{4} x^{4}+20 g_{3} x^{3}+15 g_{2} x^{2}+6 g_{1} x+g_{0}
$$

Якобиан кривой $\mathcal{V}$ есть по существу симметричное произведение $\mathrm{Sym}^{2} \mathcal{V}$, и если $\left(x_{1}, y_{1}\right)$ и $\left(x_{2}, y_{2}\right)$ - пара точек на $\mathcal{V}$, то два голоморфных дифференциала на якобиане имеют вид

$$
\begin{aligned}
& d u_{1}=\frac{d x_{1}}{y_{1}}+\frac{d x_{2}}{y_{2}} \\
& d u_{2}=\frac{x_{1} d x_{1}}{y_{1}}+\frac{x_{2} d x_{2}}{y_{2}}
\end{aligned}
$$

Легко видеть, что $d u_{1}$ и $d u_{2}$ преобразуются линейно под действием группы $S L_{2}(\mathbb{C})$. Стандартный базис $\left\{\partial_{1}, \partial_{2}\right\}$ касательного пространства к якобиану, дуальный этим один-формам, преобразуется по дуальному представлению $V^{*}$ :

$$
\left(\begin{array}{l}
\partial_{1} \\
\partial_{2}
\end{array}\right) \mapsto\left(\begin{array}{cc}
\alpha & -\beta \\
-\gamma & \delta
\end{array}\right)\left(\begin{array}{l}
\partial_{1} \\
\partial_{2}
\end{array}\right)
$$

Инфинитезимальная форма этих преобразований имеет вид

$$
\begin{array}{ll}
e\left(\partial_{1}\right)=\partial_{2}, & e\left(\partial_{2}\right)=0 \\
f\left(\partial_{1}\right)=0, & f\left(\partial_{2}\right)=\partial_{1}
\end{array}
$$


В дальнейшем мы сосредоточимся на объектах, которые являются конечномерными представлениями $S L_{2}(\mathbb{C})$. Это имеет ряд преимушеств. Во-первых, любое тождество в теории ассоциированных ю-функций будет принадлежать мультиплету тождеств, члены которого можно получить действием операторов $e$ и $f$. Эта ковариантность, с одной стороны, представляет собой удобный механизм получения этих тождеств, а с другой стороны, обеспечивает проверку их алгебраической правильности. Во-вторых, сама теория представлений подсказывает, где искать такие тождества. Действительно, она фактически предлагает естественные определения нового семейства функций ю-типа, обозначаемых здесь через $\mathcal{P}$. Наконец, при детальном рассмотрении выявляются и другие преимушества.

Однако следуюший шаг представляется шагом назад, поскольку классическая теория $\wp$-функций имеет дело не с кривой $\mathcal{V}$ в ее общей форме $(1)$, а с канонической формой

$$
Y^{2}=4 X^{5}+15 G_{4} X^{4}+20 G_{3} X^{3}+15 G_{2} X^{2}+6 G_{1} X+G_{0}
$$

обозначаемой здесь через $\pi(\mathcal{V})$. Это уравнение кривой пятого порядка получается из $(1)$ $S L_{2}(\mathbb{C})$-преобразованием с параметрами $\alpha=\mu, \beta=0, \gamma=\mu / \theta$ и $\delta=1 / \mu$, где $g(\theta)=0$,

$$
\frac{2}{3 \mu^{4}}=g_{5}+\frac{5 g_{4}}{\theta}+\frac{19 g_{3}}{\theta^{2}}+\frac{10 g_{2}}{\theta^{3}}+\frac{5 g_{1}}{\theta^{4}}+\frac{g_{0}}{\theta^{5}}
$$

$\pi$ обозначает эту специальную, использующую параметры $\theta$ и $\mu$, проекцию на каноническую форму.

Этот "выбор калибровки" нарушает ковариантность семейства кривых. Отображению $\pi$ отвечает отображение $\pi^{*}$ векторных полей $e, f$ и $h$. Аккуратное вычисление показывает, что

где

$$
\pi^{*}(e)=\frac{1}{\mu^{2}} E, \quad \pi^{*}(f)=0, \quad \pi^{*}(h)=0,
$$

$$
E=-\frac{\partial}{\partial X}+\sum_{p=0}^{3}(6-p) G_{p+1} \frac{\partial}{\partial G_{p}}+\frac{4}{3} \frac{\partial}{\partial G_{4}}
$$

Таким образом, спроектированная кривая еше "чувствует" действие векторного поля $e$, но вариации в направлениях $f$ и $h$ подавлены нормировкой коэффициентов $G_{6}=0$ и $G_{5}=2 / 3$. Это остаточное действие можно было бы устранять и далее, нормируя другие коэффициенты кривой пятого порядка (как это, вообще говоря, и делают с кубической кривой в случае первого рода), но, кажется, исторически так не делается, и с нашей точки зрения это было бы еше бо́льшим шагом назад.

Используя точки $\left(X_{1}, Y_{1}\right)$ и $\left(X_{2}, Y_{2}\right)$, определим на кривой $\pi(\mathcal{V})$ семейство функций с двойным индексом

$$
\begin{aligned}
& P_{22}=X_{1}+X_{2}+\frac{3}{2} G_{4}, \\
& P_{12}=-X_{1} X_{2}+\frac{1}{2} G_{3}, \\
& P_{11}=\frac{F\left(X_{1}, X_{2}\right)-2 Y_{1} Y_{2}}{2\left(X_{1}-X_{2}\right)^{2}}+\frac{3}{2} G_{2},
\end{aligned}
$$


где $F$ - полярная форма

$$
\begin{aligned}
F\left(x_{1}, X_{2}\right)= & 4 X_{1}^{2} X_{2}^{2}\left(X_{1}+X_{2}\right)+30 G_{4} X_{1}^{2} X_{2}^{2}+20 G_{3} X_{1} X_{2}\left(X_{1}+X_{2}\right)+ \\
& +30 X_{1} X_{2}+6 G_{1}\left(X_{1}+X_{2}\right)+2 G_{0} .
\end{aligned}
$$

Непосредственно проверяется, что $E\left(P_{11}\right)=2 P_{12}, E\left(P_{12}\right)=P_{22}$ и $E\left(P_{22}\right)=0$. Справедливы также соотношения $\partial_{1} P_{22}=\partial_{2} P_{12}, \partial_{1} P_{12}=\partial_{2} P_{11}$, и соответственно сушествует потенциал $P$ такой, что $P_{11}=\partial_{1}^{2} P, P_{12}=\partial_{1} \partial_{2} P$ и $P_{22}=\partial_{2}^{2} P$. Это согласуется с действием $E, E\left(\partial_{1}\right)=\partial_{2}, E\left(\partial_{2}\right)=0$, но это все, что мы можем сказать о действии группы, так как триплет $\left(P_{11}, P_{12}, P_{22}\right)$ не является полным представлением $S L_{2}(\mathbb{C})$. Классическое средство [3] против этого недостатка - навязать ковариантность, определив вектор из двухиндексных ю-функций при помощи преобразования, обратного преобразованию, проектирующему данную кривую на ее каноническую форму. Таким образом,

$$
\left(\begin{array}{l}
\wp_{22} \\
\wp_{12} \\
\wp_{11}
\end{array}\right)=\left(\begin{array}{ccc}
\frac{1}{\mu^{2}} & -\frac{2}{\theta} & \frac{\mu^{2}}{\theta^{2}} \\
0 & 1 & -\frac{\mu^{2}}{\theta} \\
0 & 0 & \mu^{2}
\end{array}\right)\left(\begin{array}{l}
P_{22} \\
P_{12} \\
P_{11}
\end{array}\right) .
$$

Установив действие $e$ и $f$ на параметры $\theta$ и $\mu$ из определяюших их соотношений и вспомнив, что $\pi^{*}(e)=E / \mu^{2}$, можно проверить, что $\wp_{i j}$ имеют правильные свойства трехмерного представления группы $S L_{2}(\mathbb{C})$ :

$$
\begin{array}{lll}
e\left(\wp_{11}\right)=2 \wp_{12}, & e\left(\wp_{12}\right)=\wp_{22}, & e\left(\wp_{22}\right)=0, \\
f\left(\wp_{22}\right)=0, & f\left(\wp_{12}\right)=\wp_{22}, & f\left(\wp_{22}\right)=2 \wp_{12} .
\end{array}
$$

Кроме того, функции ю $\wp_{j}$ сохраняют перекрестно-дифференциальные свойства функций $P_{i j}$. Соответствуюший потенциал запишем в виде $-\ln \sigma\left(u_{1}, u_{2}\right)$.

Структура оригинальных уравнений Бейкера, с которых мы начинали, с точки зрения теории представлений совершенно очевидна. Для обозначения представлений, соответствуюших функциям ю или коэффициентам уравнения кривой шестого порядка, будем использовать буквы с одним целочисленным нижним индексом, указывающим на размерность представления. Тогда соотношение между представлениями схематически можно представить в виде

$$
\wp_{5}+\left(\wp_{3} \otimes \wp_{3}\right)_{5}=\left(g_{7} \otimes g_{7}\right)_{5}+\left(g_{7} \otimes \wp_{3}\right)_{5},
$$

где $(A \otimes B)_{n}$ обозначает проекцию на (единственную) $n$-мерную неприводимую компоненту тензорного произведения. Это можно проверить, действуя операторами $e$ и $f$ на отдельные уравнения; в частности, можно видеть, что верхнее и нижнее уравнения принадлежат ядрам соответственно е и $f$.

Таким образом, пять уравнений Бейкера являются выражением одного тензорного тождества. Отсюда ясно также, что они порождают семейство решений уравнения Буссинеска. Например, дважды продифференцировав последнее из уравнений по $u_{2}$, введя 
обозначение $\phi=\wp_{22}$, проведя масштабное преобразование, добавив подходящую константу и выполнив галилеевский буст, мы воспроизведем уравнение Буссинеска. Это не самое общее решение, поскольку оно должно удовлетворять еше четырем другим уравнениям, но любая кривая рода 2 дает такое решение.

Это наблюдение плодотворно еше с одной стороны. Для уравнения Буссинеска существует хорошо известная пара Лакса. Но в этом случае тензорная природа уравнений Бейкера позволяет обобщить эту пару на зависящую от параметра пару Лакса, условием совместности которой является полная система пяти уравнений. Операторы Лакса - это векторные поля на якобиане (подробности можно найти в работе [2]).

Функции $\wp_{i j}$ не униформизуют кривую шестого порядка. Но они действительно униформизуют поверхность Куммера, которая представляет собой якобиан $\mathrm{Sym}^{2} \mathcal{V}$, факторизованньй по инволюции. В терминах функций $\wp_{i j}$ поверхность Куммера определяется уравнением

$$
\left|\begin{array}{cccc}
g_{6} & -3 g_{5} & 3 g_{4}+2 \wp_{22} & -g_{3}-2 \wp_{12} \\
-3 g_{5} & 9 g_{4}-4 \wp_{22} & -9 g_{3}+2 \wp_{12} & 3 g_{2}+2 \wp_{11} \\
3 g_{4}+2 \wp_{22} & -9 g_{3}+2 \wp_{12} & 9 g_{2}-4 \wp_{11} & -3 g_{1} \\
-g_{3}-2 \wp_{12} & 3 g_{2}+2 \wp_{11} & -3 g_{1} & g_{0}
\end{array}\right|=0 .
$$

Вышеприведенное определение двухиндексных ю-функций является классическим, однако оно не включает в себя основополагающую ковариантность естественным образом. В дальнейшем мы представим семейство естественно ковариантных двухиндексных объектов с теми же необходимыми свойствами. Будет показано, как легко получаются тождества для этих объектов и их производных и как эти тождества сводятся к классическим при соответствуюших условиях.

Но прежде чем заняться этим, остановимся кратко на роли отображений ХиротыБейкера (“производных") в теории представлений (детали см. в [5], [6]).

Элементы $e, f$ и $h$ алгебры $s l_{2}(\mathbb{C})$ действуют, вообше говоря, на $n$-мерных представлениях $A_{n}, B_{n}, \ldots$ группы $S L_{2}(\mathbb{C})$. Обозначим базисы этих представлений через $\left\{a_{0}, a_{2}, \ldots, a_{n-1}\right\},\left\{b_{0}, b_{2}, \ldots, b_{n-1}\right\}, \ldots$, где $a_{0}, b_{0}, \ldots \in$ ker $e-$ векторы младшего веca, а $a_{n-1}, b_{n-1}, \ldots \in \operatorname{ker} f-$ векторы старшего веса. Действие элемента $v \in s l_{2}(\mathbb{C})$ продолжается на тензорные произведения по правилу Лейбница

$$
v(a \otimes b)=v(a) \otimes b+a \otimes v(b)
$$

и т.д.

Простейшее отображение Хироты-Бейкера определяется как отображение одного тензорного произведения на другое:

$$
D: A_{n} \otimes B_{m} \rightarrow A_{n+1} \otimes B_{m+1}
$$

по формуле

$$
D\left(a_{p} \otimes b_{q}\right)=a_{p+1} \otimes b_{q}-a_{p} \otimes b_{q+1} .
$$


В этом случае (двукратного тензорного произведения) оператор $D$ является сплетающим по отношению к действию алгебры $s l_{2}(\mathbb{C})$, т.е.

$$
[D, e]=[D, h]=[D, f]=0 .
$$

В случае тензорного произведения порядка $s$ имеется $s-1$ таких (обобшенных) операторов $D$, но они являются сплетаюшими лишь частично: они коммутируют с подалгеброй, порождаемой единственным элементом $f$.

Сплетающий оператор $D$ переводит векторы старшего веса произведения $A_{n} \otimes B_{m}$ в старшие векторы произведения $A_{n+1} \otimes B_{m+1}$ (в случае тензорного произведения второго порядка это же справедливо для векторов младшего веса). Поскольку векторы старшего веса нумеруют неприводимые представления, отображение Бейкера-Хироты позволяет явно построить базисы неприводимых компонент любого тензорного произведения. Фактически полиномы по обобщенным операторам обеспечивают полное описание плетизма [6].

Заметим, что производная Хироты [4] получается из вышеописанного оператора $D$ при переходе к бесконечномерным проективным представлениям, в этом случае алгебра $s l_{2}(\mathbb{C})$ заменяется алгеброй Гейзенберга [8].

Покажем, как используется оператор $D$, на примере двумерного и трехмерного представлений $\wp_{2}$ и $\wp_{3}$ с базисами $\left\{\wp_{1}, \wp_{2}\right\}$ и $\left\{\wp_{11}, \wp_{12}, \wp_{22}\right\}$, соответственно. Вектор старшего веса имеет вид $\mathbf{v}=\wp_{2} \otimes \wp_{22}$. Поскольку $\mathbf{2} \otimes \mathbf{3}=\mathbf{2} \oplus \mathbf{4}$, этот вектор порождает неприводимое представление размерности четыре. Образ $D$ на этом векторе имеет вид

$$
D(\mathbf{v})=\wp_{22} \otimes \wp_{122}-\wp_{12} \otimes \wp_{222}
$$

и является вектором старшего веса другого неприводимого представления той же размерности четыре.

Введем семейство новых двухиндексных объектов, которые обозначим через $\mathcal{P}$. Они реализуют трехмерное представление группы $S L_{2}(\mathbb{C})$ :

$$
\mathcal{P}_{11}=\frac{2 x_{1} x_{2}}{x_{1}-x_{2}}, \quad \mathcal{P}_{12}=-\frac{x_{1}+x_{2}}{x_{1}-x_{2}}, \quad \mathcal{P}_{22}=\frac{2}{x_{1}-x_{2}} .
$$

Замечательно, что эти функции удовлетворяют условию для перекрестных производных

$$
\partial_{1} \mathcal{P}_{12}=\partial_{2} \mathcal{P}_{11}, \quad \partial_{2} \mathcal{P}_{12}=\partial_{1} \mathcal{P}_{22}
$$

и, следовательно, могут быть выражены в терминах потенциала $\mathcal{P}_{i j}=\partial_{i} \partial_{j} \mathcal{P}$, который должен удовлетворять уравнению Монжа-Ампера

$$
\mathcal{P}_{12}^{2}-\mathcal{P}_{11} \mathcal{P}_{22}=1 .
$$

Теперь у нас есть набор инструментов для построения дифференциальных тождеств, которым удовлетворяют все многоиндексные $\mathcal{P}$-функции: алгебраическая кривая $y_{i}^{2}=$ $g\left(x_{i}\right)$ для $i=1,2$, производные $\partial_{i}$ при $i=1,2$, отображаюшие $n$-мерные представления 
в $(n+1)$-мерные, и отображение $D$ Хироты-Бейкера, которое выделяет векторы старшего веса и тем самым указывает, как классифицировать тождества.

Например, из вышеприведенного трехмерного представления мы построили четырехмерное представление $\left\{\mathcal{P}_{111}, \mathcal{P}_{112}, \mathcal{P}_{122}, \mathcal{P}_{222}\right\}$. Поскольку $\mathbf{4} \otimes \mathbf{4}=\mathbf{1} \oplus \mathbf{3} \oplus \mathbf{5} \oplus \mathbf{7}$, где только $\mathbf{3}$ и $\mathbf{7}$ остаются после симметризации в полиномы, естественно обратить внимание на векторы старшего веса $\mathcal{P}_{112} \mathcal{P}_{222}-\mathcal{P}_{122}^{2}$ (происходящего из $\left.D^{2}\left(\mathcal{P}_{2} \otimes \mathcal{P}_{2}\right)\right)$ и $\mathcal{P}_{222}^{2}$, соответственно. Исходя из определения производных $\partial_{1}$ и $\partial_{2}$ находим , что трехиндексные $\mathcal{P}$-функции имеют вид

$$
\begin{array}{ll}
\mathcal{P}_{111}=2 \frac{y_{1} x_{2}^{3}+y_{2} x_{1}^{3}}{\left(x_{1}-x_{2}\right)^{3}}, & \mathcal{P}_{112}=2 \frac{y_{1} x_{2}^{2}+y_{2} x_{1}^{2}}{\left(x_{1}-x_{2}\right)^{3}}, \\
\mathcal{P}_{122}=2 \frac{y_{1} x_{2}+y_{2} x_{1}}{\left(x_{1}-x_{2}\right)^{3}}, & \mathcal{P}_{222}=2 \frac{y_{1}+y_{2}}{\left(x_{1}-x_{2}\right)^{3}} .
\end{array}
$$

Тогда из тождеств, связываюших между собой $x_{i}$ и $y_{i}$, следует, что

$$
\mathcal{P}_{222}^{2}-\mathcal{I P}_{22}^{3}=F_{6}\left(\mathcal{P}_{11}, \mathcal{P}_{12}, \mathcal{P}_{22}\right)
$$

Здесь $F_{6}$ - полином шестой степени, коэффициенты которого зависят от $g_{i}$. Его детальный вид можно найти в работе [1], но с современной точки зрения важно лишш то, что это старший вектор семимерного неприводимого представления. Величина $\mathcal{I}$ является очень важным инвариантом (представлением размерности один), к которому мы вскоре вернемся. Полный набор семи тождеств генерируется рекуррентным применением оператора $f$ до тех пор, пока не получится вектор младшего веса.

Тождества для трехмерного представления имеют более простой вид:

$$
\begin{aligned}
\mathcal{P}_{112} \mathcal{P}_{222}-\mathcal{P}_{122}^{2} & =\mathcal{I P}_{22} \\
\mathcal{P}_{111} \mathcal{P}_{222}-\mathcal{P}_{122} \mathcal{P}_{112} & =2 \mathcal{I P}_{12} \\
\mathcal{P}_{111} \mathcal{P}_{122}-\mathcal{P}_{112}^{2} & =\mathcal{I} \mathcal{P}_{11}
\end{aligned}
$$

Инвариант $\mathcal{I}$ равен $2 y_{1} y_{2} /\left(x_{1}-x_{2}\right)^{3}$, или в терминах $\mathcal{P}$-функций

$$
\mathcal{I}=\left|\begin{array}{ccc}
\mathcal{P}_{111} & \mathcal{P}_{112} & \mathcal{P}_{122} \\
\mathcal{P}_{112} & \mathcal{P}_{122} & \mathcal{P}_{222} \\
\mathcal{P}_{11} & \mathcal{P}_{12} & \mathcal{P}_{22}
\end{array}\right| .
$$

Он явно удовлетворяет квадратичному тождеству

$$
\mathcal{I}^{2}=S\left(\mathcal{P}_{11}, \mathcal{P}_{12}, \mathcal{P}_{22}\right)
$$

где функция в правой части является инвариантом кривой шестого порядка. Это соотношение симметрично при перестановке $\left(x_{1}, y_{1}\right)$ и $\left(x_{2}, y_{2}\right)$ и одновременном отождествлении инволютивных точек $y \mapsto-y$. Следовательно, оно представляет собой уравнение якобиана $\mathrm{Sym}^{2} \mathcal{V}$, факторизованного по инволюции, т.е. уравнение поверхности Куммеpa. 
В этой форме тождество имеет интересный геометрический смысл. Если записать трехмерное представление в виде $\mathbf{x}=\left(\mathcal{P}_{11}, \mathcal{P}_{12}, \mathcal{P}_{22}\right)$, то $\mathcal{I}$ оказывается скалярным тройным произведением

$$
\mathcal{I}=\mathbf{x} \cdot\left(\partial_{1} \mathbf{x} \times \partial_{2} \mathbf{x}\right),
$$

где $\mathbf{x}$ лежит на квадратичной форме, описываемой уравнением Монжа-Ампера (относительно дальнейших деталей этого подхода и теории представлений см. соответственно [1] и [2]).

Дальнейшие исследования возможны по ряду направлений. Наиболее очевидное связано с кривыми высших родов [9]. Пока мы имеем дело с кривыми вида $f(x, y)=0$, вполне вероятно, что именно теория представлений группы $S L_{2}(\mathbb{C})$ является важной. Однако возможно, что фундаментальные двухиндексные объекты нужно определять несколько по-другому и включать представления более общих размерностей. Вторая тема - это формулы сложения. Вообще говоря, они выписьваются применительно к канонической форме, поэтому ковариантность не является очевидной. Вероятно, законы абелевой группы можно сформулировать подобным образом. Наконец, классическим является вопрос о разложении функции $\sigma$ по $u_{1}$ и $u_{2}$. Опыт исследований в области систем дифференциальных уравнений, зависящих от параметров, дает возможность предположить, что рекурсивные операторы для таких рядов можно найти, если записывать их в ковариантной форме.

Благодарности. Автор и его соавторы, К. Эйлбек и В. Энольски, благодарят Институт математических наук имени Исаака Ньютона, Кембридж, Великобритания, и в частности организаторов и участников конференции Integrable Systems Programme, coстоявшейся там в конце 2002 г., за проявленный интерес и поддержку. Автор благодарен также Университету Глазго за финансовую поддержку, позволившую ему принять участие в конференции NEEDS 2002 в Кадисе.

\section{Список литературы}

[1] C. Athorne, J. J. C. Eilbeck, V. Z. Enolski. An $S L_{2}$ covariant theory of genus 2 hyperelliptic functions. Glasgow Dept. Math. Preprint. Glasgow: Glasgow Dept. Math., 2002.

[2] C. Athorne, J. J. C. Eilbeck, V. Z. Enolski. Identities for the classical genus $2 \wp$ function. Glasgow Dept. Math. Preprint. Glasgow: Glasgow Dept. Math., 2002.

[3] H. F. Baker. Multiply Periodic Functions. Cambridge: Cambridge Univ. Press, 1907.

[4] R. Hirota. Direct method of finding exact solutions of nonlinear evolution equations. In: Bäcklund Transformations, the Inverse Scattering Method, Solitons, and Their Applications. Lect. Notes Math. V. 515. Ed. R. M. Miura. Berlin: Springer, 1976. P. 40-68.

[5] C. Athorne. Phys. Lett. A. 1999. V. 256. P. 20-24.

[6] C. Athorne. Glasgow Math. J. 2001. P. 1-8.

[7] B. Grammaticos, A. Ramani, J. Hietarinta. Phys. Lett. A. 1994. V. 190. P. 65-70.

[8] P. J. Olver, J. A. Sanders. Adv. Appl. Math. 2000. V. 25. P. 252-283.

[9] V. M. Buchstaber, V.Z. Enolski'i, D. V. Leykin. Rev. Math. Phys. 1997. V. 10. P. 3-120. 